108

FUTURE CARDIOVASCULAR DISEASE RISK FOR WOMEN WITH A HISTORY OF GESTATIONAL HYPERTENSION: A SYSTEMATIC REVIEW AND META-ANALYSIS

${ }^{1}$ Clare Oliver-Williams*, ${ }^{2}$ Charmaine Lo, ${ }^{1}$ Andre Lo, ${ }^{1}$ Shu Hui, ${ }^{3}$ Grace Fisher, ${ }^{1}$ Beth Corker, ${ }^{1}$ Olivia Batho, ${ }^{1}$ Bethan Morris, ${ }^{1}$ Monika Chowaniec, ${ }^{4}$ Abigail Fraser. ${ }^{1}$ University of Cambridge; ${ }^{2}$ University of Newcastle Australia; ${ }^{3}$ Swavesey College; ${ }^{4}$ University of Bristol

\subsection{6/heartjnl-2019-BCS.105}

Introduction The risk of cardiovascular outcomes associated with gestational hypertension is unclear. This study aimed to quantify the association between gestational hypertension and cardiovascular outcomes for women.

Design Systematic review and meta-analysis.

Data Sources

PubMed, Embase and Web of Science.

Eligibility Criteria

Studies examining the association between gestational hypertension and any cardiovascular outcome, including cardiovascular disease, coronary heart disease, stroke and heart failure. Two reviewers independently assessed the abstracts and full-text articles. Study characteristics and the relative risk of cardiovascular outcomes associated with gestational hypertension were extracted from eligible studies. Where appropriate, estimates were pooled with inverse variance weighted randomeffects meta-analysis, and the absolute risk increases were calculated using the European population as a reference, as the majority of studies came from Europe.

Results Nineteen studies involving 3,601,192 women (128,445 with gestational hypertension) were identified. A history of one or more pregnancies affected by gestational hypertension was associated with an increased risk of cardiovascular disease (12 studies, relative risk 1.73, 95\% confidence interval: 1.43-2.08), coronary heart disease (8 studies, $1.56,1.35-1.81)$ and heart failure (4 studies, 1.70, 1.432.02). (See Figure) There was also evidence for an increased risk of stroke (9 studies, 1.66, 0.99-2.80). Among the

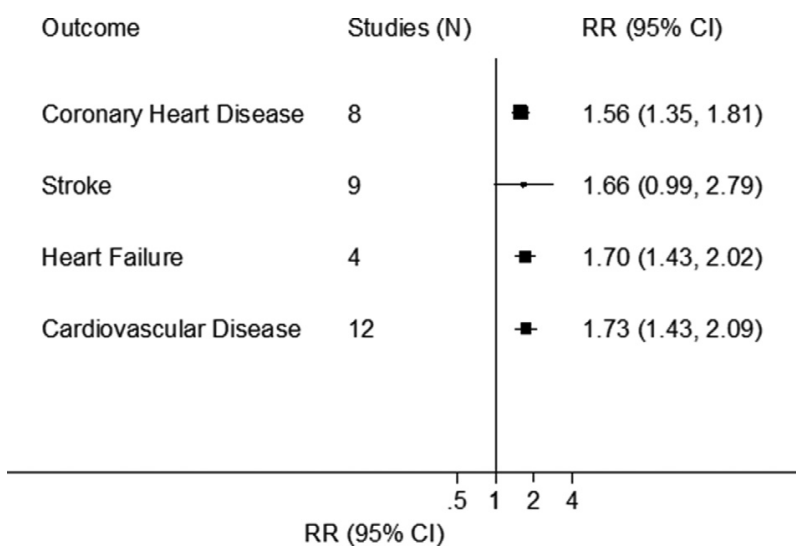

Abstract 108 Figure 1 Association between gestational hypertension and cardiovascular outcomes, showing summary relative risks for each outcome examined outcomes examined, the highest absolute risk increase was for cardiovascular disease: 14.0 events/1000 person-years. Associations between gestational hypertension and cardiovascular disease were broadly consistent across subgroups, although there was evidence that high quality studies with a low risk of bias had lower effect estimates. When analyses were restricted to high quality studies, an increased risk was found for all outcomes: cardiovascular disease, (1.53, 1.25$1.88)$; coronary heart disease, $(1.40,1.26-1.54)$; stroke, $(1.35,1.14-1.60)$; and heart failure, $(1.70,1.43-2.02)$.

Conclusion Gestational hypertension is associated with an increased risk of overall cardiovascular disease, coronary heart disease, stroke and heart failure. Only two studies evaluated risk associated with the number of pregnancies affected by gestational hypertension, therefore more research is needed to assess the presence of a dose-response relationship.

Conflict of Interest None

\section{ESC RISK SCORE-ADJUSTED COST ANALYSIS OF THE INVESTIGATIONS IN STABLE CHEST PAIN: NICE VS. ESC GUIDELINES}

${ }^{1}$ Alexandros Papachristidis*, ${ }^{2}$ Sarah Denny, ${ }^{3}$ George Vaughan, ${ }^{1}$ Tamim Akbari, ${ }^{2}$ Edith Avornyo, ${ }^{1}$ Tracey Griffiths, ${ }^{1}$ Emma Saunders, 'Jonathan Byrne, 'Mark Monaghan, ${ }^{3}$ Khaled Alfakih. ${ }^{1}$ King's College Hospital; ${ }^{2}$ Lewisham and Greenwich NHS Foundation Trust; ${ }^{3}$ King's College London

\subsection{6/heartjnl-2019-BCS.106}

Introduction National Institute for Health and Clinical Excellence (NICE) have removed the use of pre-test probability risk score (RS) in patients with new onset stable chest pain. They recommend computed tomography coronary angiography (CTCA) as first line investigation irrespective of RS. European Society of Cardiology (ESC) suggest using the ESC RS and recommend functional tests as initial investigation in patients with RS $15-85 \%$ and allow for the use of CTCA in patients with RS of $15-50 \%$. We compare the two recommended strategies (NICE vs ESC) as applied in two neighbouring NHS Trusts in South London. We additionally investigate the prognostic role of ESC RS in terms of need for revascularization.

Methods Two groups of patients, who attended rapid access chest pain clinics in two neighbouring NHS Trusts were recruited. Group A $(\mathrm{N}=667)$ were investigated based on ESC guidelines, whereas Group B $(\mathrm{N}=654)$ were investigated following NICE guidance. The RS was calculated as per ESC recommendation based on patient age, gender and typicality of chest pain. The patients were divided in two subgroups according to ESC RS. Sub-groups A1 and B1, were of patients with lower RS (15-50\%) and sub-groups A2 and B2 were of patients with higher RS (>50\%). The need for invasive coronary angiography (ICA) and revascularization were compered between groups and sub-groups. A cost analysis was performed based on UK tariffs for CTCA $(£ 220)$, stress echo $(£ 176)$ and ICA $(£ 1,001)$. 


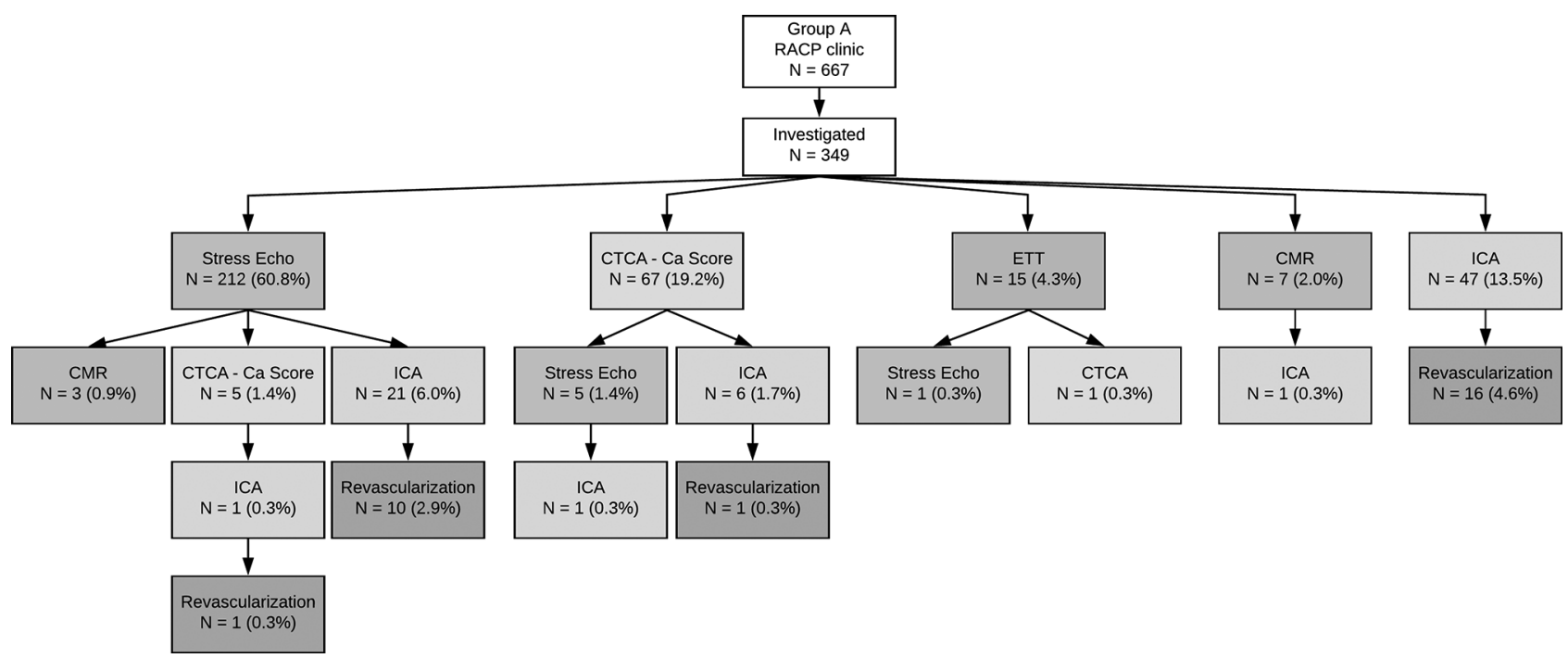

Abstract 109 Figure 1

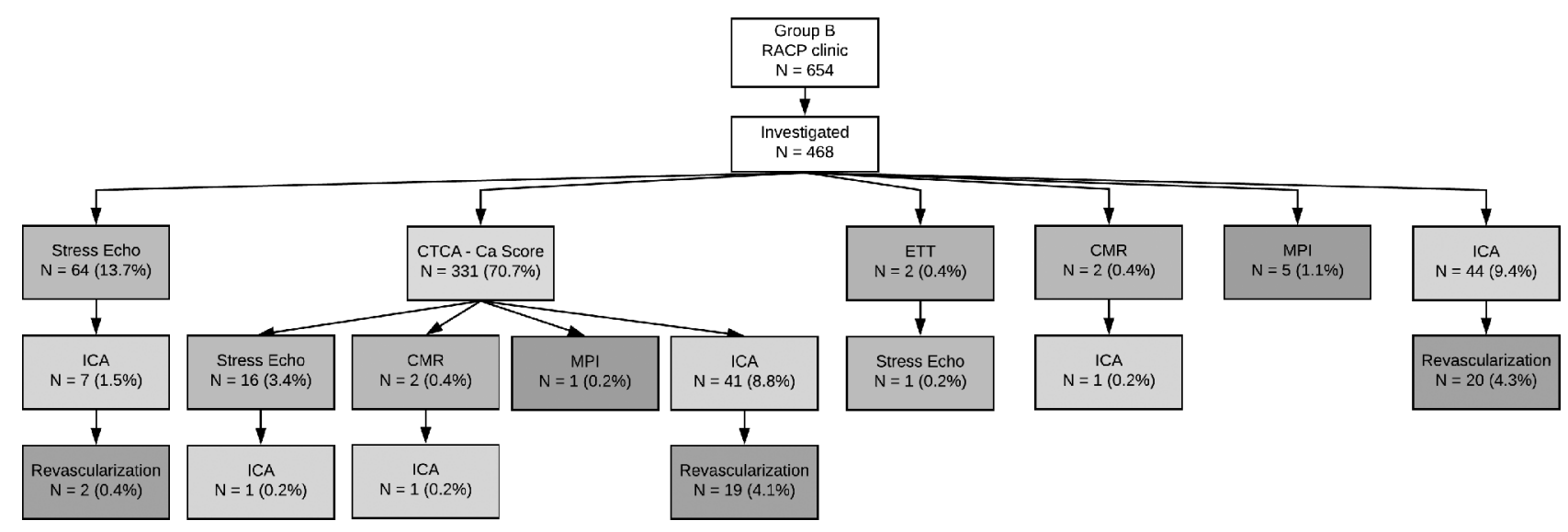

Abstract 109 Figure 2

Abstract 109 Table 1

\begin{tabular}{|l|c|c|c|c|}
\hline & Group A & Group B & p value & All \\
\hline Age & $57.2 \pm 12.2$ & $56.9 \pm 10.9$ & 0.697 & $57.0 \pm 11.5$ \\
\hline Gender (male) & $174(49.9 \%)$ & $240(51.3 \%)$ & 0.687 & $414(50.7 \%)$ \\
\hline ESC Risk Score & $39.2 .6 \pm 21.3$ & $41.12 \pm 21.2$ & 0.214 & $36.1 \pm 21.0$ \\
\hline Hypertension & $154(44.1 \%)$ & $199(42.5 \%)$ & 0.705 & $353(43.2 \%)$ \\
\hline Hypercholesterolaemia & $216(61.9 \%)$ & $264(56.4 \%)$ & 0.142 & $480(58.8 \%)$ \\
\hline Diabetes & $79(22.6 \%)$ & $94(20.1 \%)$ & 0.403 & $173(21.2 \%)$ \\
\hline Family History & $149(42.7 \%)$ & $184(39.3 \%)$ & 0.370 & $333(40.8 \%)$ \\
\hline Smoking & $80(22.9 \%)$ & $88(18.8 \%)$ & 0.168 & $168(20.6 \%)$ \\
\hline ICA & $77(22.1 \%)$ & $95(20.3 \%)$ & 0.608 & $172(21.1 \%)$ \\
\hline Revascularization & $28(8.0 \%)$ & $41(8.8 \%)$ & 0.708 & $69(8.4 \%)$ \\
\hline
\end{tabular}


Results Baseline characteristics and the prevalence of cardiovascular risk factors were similar between Groups A and B (Table 1). The rate of progression to ICA was comparable in the two groups $(9.9 \%$ vs $12.0 \% ; \mathrm{p}=0.377)$, as was the rate of revascularization $(4.0 \%$ vs $5.0 \% ; \mathrm{p}=0.532)$ (Figure 1$)$. The average per investigated patient cost was lower in Group A by $£ 46.11$ ( $£ 279.66$ vs $£ 325.77$ ).

In Group A1 there was a lower rate of progression to ICA compared to Group B1 $(8.7 \%$ vs $12.6 \%, \mathrm{p}=0.177)$ as was for revascularization $(2.6 \%$ vs $5.5 \%, \mathrm{p}=0.122)$. The average per patient cost was considerably lower in Group A1 by $£ 69.54$.

$18.5 \%$ of patients proceeded to ICA in Group A2 compared to $14.6 \%$ in Group B2 $(\mathrm{p}=0.512)$ and $10.8 \%$ were revascularized as opposed to $5.2 \%(\mathrm{p}=0.187)$. The average cost per investigated patient was slightly higher in Group A2 by $£ 20.99$.

Investigating possible predictors of revascularization (ESC RS, diabetes, family history of coronary artery disease, smoking, hypercholesterolaemia), only the ESC RS was found to be independently related to the need for revascularization (OR: 1.049, 95\%CI: 1.036-1.062, $\mathrm{p}<0.001)$.

Conclusion Both NICE and ESC recommendations on new onset stable chest pain lead to similar rates of progression to ICA and revascularization, but lower cost when stress echo (ESC guidance) is used as first line investigation. There was no significant difference when we repeated the analysis in low and high ESC RS patients. However, the ESC RS was the only independent predictor of need for revascularization.

Conflict of Interest None

\section{CORNEAL BIOMECHANICAL PROPERTIES AND VASCULAR COMPLIANCE IN THE UK BIOBANK COHORT}

${ }^{1}$ Simon Woodbridge*, ${ }^{2}$ Swan Kang, ${ }^{3}$ Nay Aung, ${ }^{4}$ Luca Biasiolli, ${ }^{5}$ Jackie Cooper, ${ }^{3}$ Mihir Sanghvi, ${ }^{5}$ Kenneth Fung, ${ }^{4}$ Stefan Piechnik, ${ }^{6}$ Stefan Neubauer, ${ }^{5}$ Steffen Petersen, ${ }^{7}$ Paul Foster. ${ }^{1}$ William Harvey Research Institute; ${ }^{2}$ Moorfields Eye Hospital NHS Foundation Trust, London, UK; ${ }^{3}$ William Harvey Research Institute, Queen Mary University of London; ${ }^{4}$ Division of Cardiovascular Medicine, University of Oxford; ${ }^{5}$ William Harvey Research Institute, NIHR Barts Biomedical Research Centre, London, UK; ${ }^{6}$ Oxford University; ${ }^{7}$ NIHR Biomedical Research Centre, Moorfields Eye Hospital NHS Foundation Trust and UCL Institute of Ophthalmology, London

\subsection{6/heartjnl-2019-BCS.107}

Introduction Intra-ocular pressure (IOP) measurement is an integral part a comprehensive eye examination. In addition to IOP, corneal biomechanical characteristics such as corneal hysteresis $(\mathrm{CH})$, a measurement of viscoelastic compliance, and corneal resistance factor (CRF), derived from corneal deformability, have also been identified as useful indicators of incidence and progression of primary open angle glaucoma (POAG) $(1,2)$. Corneal tissue shares compositionally similar properties with arterial tissue $(3,4)$. Our cross-sectional observational study aimed to ascertain whether corneal biomechanical metrics $(\mathrm{CH} \&$ CRF) are associated with arterial stiffness - a well-established marker of future cardiovascular (CV) events and mortality.

Methods From an initial pool of 5065 participants from the community-based UK Biobank study, 4018 were rejected for missing data, leaving a cohort of 1047 individuals (male/female ratio: 0.496, mean age: 62 years, white ethnicity: 96.1\%) (Table 1). Corneal biomechanical metrics ( $\mathrm{CH} \& \mathrm{CRF}$ ), were obtained using a Reichert Ocular Response Analyzer (ORA). Arterial compliance was quantified by aortic distensibility (AoD) derived by cardiovascular magnetic resonance (CMR) imaging. The relationship between corneal and vascular compliance parameters was assessed using both Spearman rank correlation coefficient analysis, and univariable and multivariable regression analyses adjusting for potential influential confounding variables - age, sex, ethnicity, height, weight, systolic blood pressure (SBP), diastolic blood pressure (DBP), smoking status, regular alcohol intake, diabetes status and dyslipidaemia.

Results A significant weakly positive correlation was observed between $\mathrm{CH}$ and $\mathrm{AoD}$ at both the ascending aorta (AA) and proximal descending aorta (PDA) (AA: Rho $=0.08, \mathrm{p}=0.01 ;$ PDA: Rho $=0.11, \mathrm{p}<0.01)$, however no significant correlation was observed between CRF and AoD.

In univariable analysis, only $\mathrm{CH}$ produced significant changes in AoD at both the AA and PDA (AA: $B=+3.0 \%$ per $10 \%$ increase in $\mathrm{CH}, 95 \% \mathrm{CI}=0.6$ to $5.5, \mathrm{p}=0.02$; PDA: $\beta=+2.6 \%$ per $10 \%$ increase in $\mathrm{CH}, 95 \% \mathrm{CI}=0.8$ to $4.4, p=0.004$ ) (Figure 1 ). There was no significant linear relationship between $\mathrm{CH}$ or $\mathrm{CRF}$ and $\mathrm{AoD}$ in multivariable regression analysis, at both the $\mathrm{AA}$ and $\mathrm{PDA}(\mathrm{CH}$ at $\mathrm{AA}: \mathrm{B}=+0.8 \%$ per $10 \%$ increase in $\mathrm{CH}, 95 \% \mathrm{CI}=-0.9$ to 2.6, $\mathrm{p}=0.37 ; \mathrm{CH}$ at $\mathrm{PDA}: \beta=+0.8 \%$ per $10 \%$ increase in $\mathrm{CH}, 95 \% \mathrm{CI}=-0.3$ to $2.0, \mathrm{p}=0.16$; $\mathrm{CRF}$ at $\mathrm{AA}: ß=+1.3 \%$ per $10 \%$ increase in $\mathrm{CH}, 95 \% \mathrm{CI}=-0.4$ to $3.0, p=0.13$; $\mathrm{CRF}$ at $\mathrm{PDA}: \beta=+0.9 \%$ per $10 \%$ increase in $\mathrm{CH}, 95 \% \mathrm{CI}=-0.3$ to $2.0, \mathrm{p}=0.13$ ).

Conclusion In this community-based cohort, we observed a weakly significant general correlation between $\mathrm{CH}$ and AoD. After adjustment for potential confounding factors, we then observed no significant relationship between corneal and aortic biomechanical indices, suggesting that in a general population, biomechanical corneal indices are not independently associated with parameters of central arterial compliance.

Conflict of Interest None 